\title{
Multiple Roles for Slits in the Control of Cell Migration in the Rostral Migratory Stream
}

\author{
Kim T. Nguyen-Ba-Charvet, ${ }^{1}$ Nathalie Picard-Riera, ${ }^{3}$ Marc Tessier-Lavigne, ${ }^{2}$ Anne Baron-Van Evercooren, ${ }^{3}$ \\ Constantino Sotelo, ${ }^{1}$ and Alain Chédotal ${ }^{1}$ \\ ${ }^{1}$ Institut National de la Santé et de la Recherche Médicale (INSERM) U106, Bâtiment de Pédiatrie, Hôpital de la Salpêtrière, 75013 Paris, France, \\ ${ }^{2}$ Department of Biological Sciences, Howard Hughes Medical Institute, Stanford University, Stanford, California 94305-5020, and ${ }^{3}$ INSERM U546, Centre \\ Hospitalier Universitaire Pitie-Salpêtrière, 75013, Paris, France
}

The subventricular zone (SVZ) contains undifferentiated cells, which proliferate and generate olfactory bulb (OB) interneurons. Throughout life, these cells leave the SVZ and migrate along the rostral migratory stream (RMS) to the OB where they differentiate. In vitro, the septum and the choroid plexus (CP) secrete repulsive factors that could orient the migration of OB precursors. Slit1 and Slit2, two known chemorepellents for developing axons, can mimic this effect. We show here that the Slit receptors Robo2 and Robo3/Rig-1 are expressed in the SVZ and the RMS and that Slit1 and Slit2 are still present in the adult septum. Using Slit1/2-deficient mice, we found that Slit1 and Slit2 are responsible for both the septum and the CP repulsive activity in vitro. In adult mice lacking Slit1, small chains of SVZ-derived cells migrate caudally into the corpus callosum, supporting a role for Slits in orienting the migration of SVZ cells. Surprisingly, in adult mice, Slit1 was also expressed by type A and type C cells in the SVZ and RMS, suggesting that Slit1 could act cell autonomously. This hypothesis was tested using cultures of SVZ explants or isolated neurospheres from Slit1 - / - or Slit1 +/- mice. In both types of cultures, the migration of SVZ cells was altered in the absence of Slit1. This suggests that the regulation of the migration of OB precursors by Slit proteins is complex and not limited to repulsion.

Key words: migration; Slit; septum; choroid plexus; Robo; SVZ

\section{Introduction}

In the developing CNS, neuronal precursors migrate sometimes over long distances to reach their final destination where they differentiate into mature functional neurons. In adult mammals, this process persists in two brain regions, the hippocampal dentate gyrus and the subventricular zone (SVZ) lining the wall of the lateral ventricles (Gage et al., 1995; Alvarez-Buylla and GarciaVerdugo, 2002). In mice, olfactory interneurons progenitors are produced postnatally from the anterior telencephalic SVZ where they are separated from the ventricle cavity by ependymal cells (Lois and Alvarez-Buylla, 1993, 1994; Luskin, 1993; Doetsch et al., 1999a). In the SVZ, specialized astrocytes bearing cilia (type B cells) generate rapidly dividing type $\mathrm{C}$ precursor cells, which give rise to neuroblasts (type A cells) destined for the olfactory bulb.

\footnotetext{
Received 0ct. 20, 2003; revised Dec. 15, 2003; accepted Dec. 23, 2003.

This work was supported by Institut National de la Santé et de la Recherche Médicale and a grant from the European Union, CEE QLK3-CT-1999-00894 to A.C., C.S., and K.N.-B.-C. by the French Ministry for Research (ACI number 5116) and "Association Pour la Recherche sur le Cancer" to A.C. K.N.-B.-C. was funded by the Fondation de France and the Fondation de la Recherche Médicale (FRM). N.P.-R. was funded by the FRM. We thank G. Rougon for the mCD24 and PSA-NCAM antibodies, S. Carroll for the Dlx2 antibody, A. Alvarez-Buylla for sharing results before publication, and V. Marillat and C. Moreau-Fauvarque for help with the in situ hybridization.

Correspondence should be addressed to A. Chédotal at his present address: Centre National de la Recherche Scientifique, Unité Mixte de Recherche 7102, Université PM Curie, case 12, 9 quai Saint-Bernard, 75005 Paris, France. E-mail: chedota@infobiogen.fr.

K. Nguyen-Ba-Charvet's and C. Sotelo's, present address: Centre National de la Recherche Scientifique Unité Mixte de Recherche 7102, Université PM Curie, 9 quai Saint-Bernard, 75005 Paris, France.

DOI:10.1523/JNEUROSCI.4729-03.2004

Copyright $\odot 2004$ Society for Neuroscience $\quad$ 0270-6474/04/241497-10\$15.00/0
}

These neuroblasts migrate tangentially along the wall of the lateral ventricle before entering the rostral migratory stream (RMS), which extends from the caudal septum to the olfactory bulb. Within the RMS, neuroblasts migrate in chains through glial tunnels (Doetsch and Alvarez-Buylla, 1996; Jankovski and Sotelo, 1996; Lois et al., 1996; Wichterle et al., 1997).

Work conducted over the last few years started unraveling the signals that direct the rostral migration of neuroblasts from the SVZ to the olfactory bulb. For instance, the polysialylated neural cell adhesion molecule (PSA-NCAM) facilitates chain migration (Hu et al., 1996; Hu, 2000), and EphB2/ephrin-B2 signaling is involved in the migration of neuroblasts in the adult SVZ (Conover et al., 2000). The secreted protein Netrin-1 and its receptor, DCC, have been proposed to orient the migration of $\mathrm{OB}$ precursors while some integrins provide traction for cellular translocation (Murase and Horwitz, 2002). More recently, it has been shown that Reelin acts as a detachment signal for olfactory precursors in the RMS after reaching the olfactory bulb (Hack et al., 2002).

Slits are diffusible proteins that are considered to participate to multiple aspects of neural development, such as axon guidance (Brose et al., 1999; Nguyen Ba-Charvet et al., 1999, 2002; Plump et al., 2002) or neuronal migration (Zhu et al., 1999; Marin et al., 2003). A diffusible activity produced by the caudal septum repels SVZ neuroblasts in cultures (Hu and Rutishauser, 1996) and Slit1 and Slit2 which are expressed in the septum mimic this effect (Hu, 1999; Wu et al., 1999). However, the precise function of Slits 
and Robos in the migration of SVZ neuroblasts is still debated, and in vivo evidence is lacking.

We show here that the septum-derived chemorepulsive factor is a combination of Slit 1 and Slit 2 and that the receptors probably involved are Robo2 and Robo3/Rig-1. Moreover, the phenotypic analysis of Slit1-deficient mice supports a role for Slits in orienting the migration of SVZ cells. We also found that Slit1 is highly expressed by SVZ cells, migrating neuroblasts (type A cells), and cells migrating from SVZ neurospheres, and that their migration is abnormal in vitro in the absence of Slit1. This suggests that Slit1 may have a paracrine or autocrine action on migrating $\mathrm{OB}$ precursors.

\section{Materials and Methods}

Animals. Swiss mice (Janvier, Le Genest Saint Isle, France) were used for in situ hybridization and in vitro studies. Slit-deficient mice were generated and genotyped by PCR as previously described (Plump et al., 2002). Actin-green fluorescent protein (GFP) mice and embryos (strain TgN(GFPU)5Nagy; The Jackson Laboratory, Bar Harbor, ME; Hadjantonakis et al., 1998) were selected using a MZFLIII fluorescence stereomicroscope equipped with a GFP filter (Leica Microsystems, RueilMalmaison, France). The day of the vaginal plug was counted as embryonic day 0 (E0), and the day of the birth as postnatal day 0 (P0). Mice were anesthetized with chloral hydrate $(350 \mathrm{mg} / \mathrm{kg})$. All animal procedures were performed in accordance with institutional guidelines.

In situ hybridization. Tissue sections were proceeded as described previously (Marillat et al., 2002).

BrdU injections and staining. We used two different protocols: (1) a short survival protocol to label rapidly dividing cells. The animals were perfused $3 \mathrm{hr}$ after a single intraperitoneal injection of BrdU (Sigma, St. Louis, MO; $15 \mathrm{mg} / \mathrm{ml}, 50 \mathrm{mg} / \mathrm{kg}$ of body weight) diluted in a saline solution $(\mathrm{NaCl} 0.9 \%, 0.007 \mathrm{~N} \mathrm{NaOH})$. Brains were cut in serial frontal sections with a freezing microtome $(30 \mu \mathrm{m})$. Seven levels were selected, and six serial sections per level were analyzed. Comparative analyses were performed on four wild-type (WT), 7 Slit1+/-, and 10 Slit1-/- mice. (2) A longer survival protocol to label slowly dividing cells (Doetsch et al., 1999b): BrdU (1 mg/ml) was given to four WT, 15 Slit1+/-, and 9 Slit 1-/ - mice in drinking water for 2 weeks followed by 1 week without BrdU. Animals were perfused as described above. Brains were cut in serial sagittal sections with a freezing microtome $(30 \mu \mathrm{m})$. Twenty sections from each brain were analyzed. Staining was performed by immunocytochemistry after a 30 min treatment in $2 \mathrm{~N} \mathrm{HCl}$ at $37^{\circ} \mathrm{C}$, using an antiBrdU antibody (1:100; Harlan, Loughborough, UK).

Immunocytochemistry. Mice were perfused transcardially with $4 \%$ paraformaldehyde in $0.1 \mathrm{~m}$ phosphate buffer, $\mathrm{pH} 7.4$ (PFA), postfixed for $3 \mathrm{hr}$, and then cryoprotected in $30 \%$ sucrose. Thirty micrometer sections were cut on a freezing microtome, blocked in PBS containing $0.2 \%$ gelatin and $0.25 \%$ Triton X-100 (except where noted), and incubated overnight at room temperature (RT) with PSA-NCAM (1:1000), monoclonal GFAP (1:400; Chemicon, Temecula, CA), mCD24 (1:1000 without Triton X-100), Ki67 (1:1000; Novocastra, Newcastle, UK), Dlx2 (1:100; gift from Sean Carroll, University of Wisconsin, Madison, WI), or anti-GFP (Molecular Probes, Eugene, OR), followed by species-specific secondary antibodies directly conjugated to fluorophores (Jackson ImmunoResearch, West Grove, PA). Sections were examined under a fluorescent microscope (DMR; Leica, Nussloch, Germany) or using a confocal microscope (TCS; Leica).

Collagen coculture assays. SVZ explants were excised from $300 \mu \mathrm{m}$ slices of P3-P6 actin-GFP or Slit1-deficient mice brains. The caudal part of the septum and the choroid plexus was taken from E15-E18 Slitdeficient mice. The explants were embedded into collagen gel as previously described (Hu and Rutishauser, 1996). They were cultured under $5 \% \mathrm{CO}_{2}$ in DMEM-F-12 supplemented with 5\% fetal calf serum, 5\% horse serum (all from Invitrogen, Carlsbad, CA) and supplemented with $100 \mathrm{ng} / \mathrm{ml}$ of heparin (Sigma). After $36 \mathrm{hr}$, cocultures were fixed $1 \mathrm{hr}$ in $4 \%$ PFA. Cell nuclei in explants were stained with a solution of $10 \mu \mathrm{g} / \mathrm{ml}$ of Hoechst number 33258 (Sigma) in PBS, 0.1\% Triton X-100 for $30 \mathrm{~min}$, and rinsed three times in PBS.
SVZ explants and cell migration assay. SVZ explants from P4-P5 Slit1deficient mice were cultured in collagen as described above or in matrigel (Becton Dickinson, Bedford, MA) in Neurobasal medium supplemented with B27 (Invitrogen). After $4 \mathrm{~d}$ in culture, images of stained explants were collected with a video camera (Photometrics CoolSnap; Roper Scientific, Inc. Trenton, NJ) digitized, and analyzed using image-processing software (Metaview; Universal Imaging Corporation, Downington, PA). Migration distance was calculated as described in Belvindrah et al. (2002). Briefly, for each explant, 24 measurements from the edge of the explant and the border of the cell migration front were performed regularly distributed around the explant. Eighteen explants were analyzed per condition. The significance was calculated by ANOVA (Statview; Abacus Concepts, Berkeley, CA).

Neurospheres. Culture media were obtained from Invitrogen, all other products were purchased from Sigma, unless specified otherwise. Cerebral hemispheres of P0-P2 from homozygous and heterozygous Slit1deficient mice were minced in HBSS supplemented with $100 \mathrm{U} / \mathrm{ml}$ penicillin, $100 \mu \mathrm{g} / \mathrm{ml}$ streptomycin, $0.15 \%$ sodium bicarbonate, and $10 \mathrm{~mm}$ HEPES buffer. Cells were then submitted to enzymatic dissociation in a solution of HBSS containing trypsin $(0.0025 \%)$. After $15 \mathrm{~min}$ at $37^{\circ} \mathrm{C}$, enzymatic dissociation was stopped with fetal calf serum, followed by mechanical dissociation through nylon nets of different mesh size (63 and $25 \mu \mathrm{m}$ ). After centrifugation at $4^{\circ} \mathrm{C}$ for $15 \mathrm{~min}$, cells were collected, washed with HBSS medium, and centrifuged $5 \mathrm{~min}$ at $1500 \mathrm{rpm}$ twice. Cells were finally collected and resuspended in a chemically defined medium called NEF constituted of DMEM-F-12 medium containing glucose $(0.6 \%)$, insulin $(25 \mu \mathrm{g} / \mathrm{ml})$, transferrin $(100 \mu \mathrm{g} / \mathrm{ml})$, putrescin $(100$ $\mu \mathrm{M})$, sodium selenite (30 nM), progesterone (400 nM), heparin $(2.5 \mu \mathrm{g} /$ $\mathrm{ml})$, cyfloxacine (2 mg), FGF-2 (20 ng/ml), and EGF (20 ng/ml).

Neurospheres migration assay. After $10-15 \mathrm{~d}$ in vitro in NEF medium, the cell migration assay was performed according to the previously described procedure (Decker et al., 2000). Briefly, the non-adherent aggregated cells or spheres, purified from the $\mathrm{P} 0-\mathrm{P} 3$ mice brains, were seeded in $5 \mu \mathrm{l}$ droplets on polyornithine $(100 \mu \mathrm{g} / \mathrm{ml})$-collagen $(2 \mathrm{mg} / \mathrm{ml})$ substrated glass. After $20 \mathrm{~min}$ of adhesion, the medium was adjusted to $500 \mu \mathrm{l}$ per well with either the NEF medium or the N medium (NEF without EGF and FGF-2). Migrating cells were followed for a $5 \mathrm{~d}$ period. At several time points ( $4 \mathrm{hr}, 24 \mathrm{hr}, 5 \mathrm{~d}$ ), the longest distance between the edge of a sphere and the leading edge of the outgrowth was measured in each quarter using an Olympus inverted microscope. At least 10 spheres were followed for each time point, and the experiment was performed in triplicate from distinct cell purification. The distances of migration were averaged between experiments, and results expressed as the mean \pm SEM. Statistical significance was assessed by the Student's $t$ test.

\section{Results}

\section{Absence of chemorepulsive activity from the septum of Slit1/ Slit2 null mutant mice}

Slits have been proposed to mediate the repulsive activity exerted by the septum on migrating SVZ-derived neuroblasts in diffusion assays (Hu, 1999; Wu et al., 1999). However, this conclusion was based on indirect evidence. To further characterize the repulsive activity of Slit, we cultured SVZ explants from P3-P6 actin-GFP transgenic mice (Hadjantonakis et al., 1998) in collagen facing septum explants from E15-E18 wild-type mice, Slit1-/- mice, Slit2-/ - mice, or compound heterozygous or homozygous Slit1/2-deficient mice. We recently used a similar approach to prove that Slits are the septum repellents for lateral olfactory tract axons (Nguyen-Ba-Charvet et al., 2002). Neuroblasts migrating from the SVZ explants were easily visualized either directly by observing GFP expression or after immunostaining with antiGFP. As previously described (Hu and Rutishauser, 1996), SVZ cells migrated away from wild-type septum explants, being almost absent from the region of the gel separating the two explants (Fig. 1A) (0.496 $\pm 0.052, \mathrm{P} / \mathrm{D}$ ratio $\pm \mathrm{SEM}$ from five independent experiments). Septum repulsive activity was not significantly different with septum explants dissected from Slit1 or Slit2 


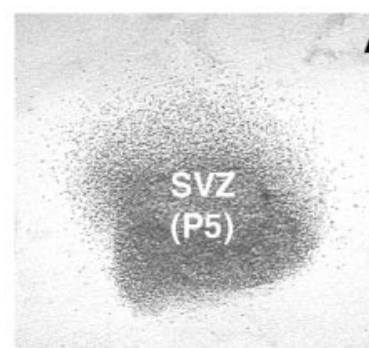

(E15) wt septum

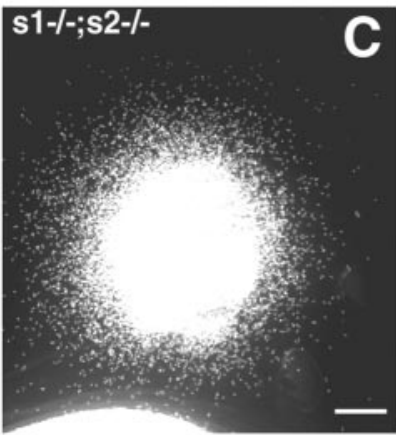

A
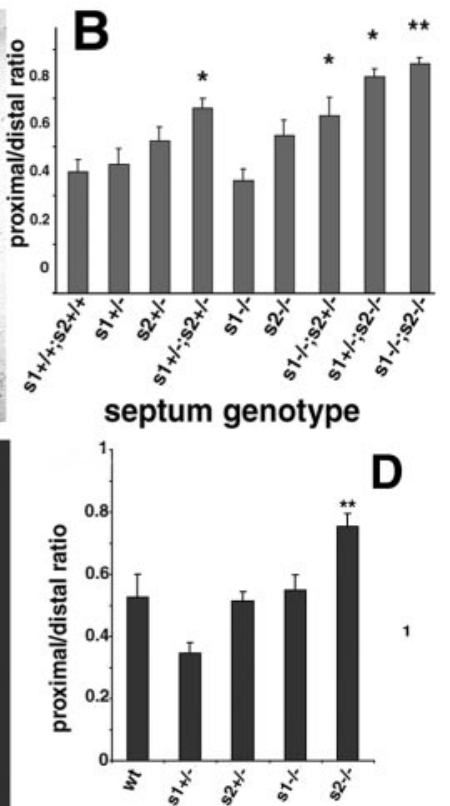

choroid plexus genotype
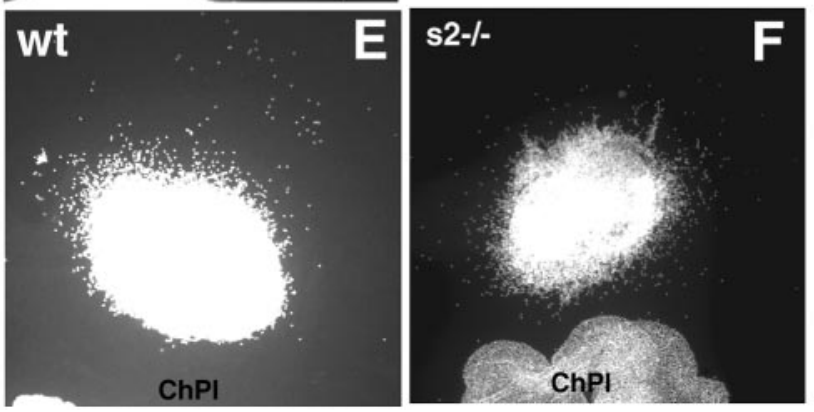

Figure 1. Slits are the septum and choroid plexus repellents for SVZ cells. SVZ explants from P4-P6GFP transgenic mice were cocultured with caudal septum ( $A-C$ ) or choroid plexus ( $\mathrm{ChPl}$, $D-F)$ explants from wild-type $(A, E)$, Slit2 $-1-(F)$, or Slit1- - ; Slit2- $-(C)$ mice. $B$, The septum repulsive activity corresponds to the ratio of the number of migrating cells in the proximal and distal quadrant. $D$, The choroid plexus repulsive activity is measured as in $B$. SEM for each condition is indicated with a bar. ${ }^{*} p<0.05 ;{ }^{* *} p<00.01$. Scale bar, $100 \mu \mathrm{m}$.

single heterozygotes $(0.652 \pm 0.089$ and $0.61 \pm 0.056$, respectively) (Fig. $1 B$ ), from single Slit $1-/-$ mice $(0.460 \pm 0.051)$ or Slit2 $-/-$ mice $(0.640 \pm 0.058)$. In contrast, when at least one allele from each gene were mutants, the repulsive activity was significantly abolished (Fig. 1 B) (Slit1+/-; Slit2+/-: $0.744 \pm$ 0.035; Slit1-/-; Slit2+/-/: $0.73 \pm 0.076$; Slit1+/-; Slit2-/-: $0.89 \pm 0.029 ; p<0.05)$. Finally, when confronted with septum from Slit1/2-deficient mice, SVZ cells migrated radially (Fig. $1 C$ ) $(0.941 \pm 2.7 ; p<0.01)$. Thus, the septum-derived chemorepulsive factor is a combination of Slit1 and Slit2.

\section{Absence of chemorepulsive activity from the choroid plexus of Slit2-/- mice}

The choroid plexus (CP) isolated from the telencephalic ventricles has also been shown to secrete repellents for olfactory bulb precursors ( $\mathrm{Hu}, 1999)$. The purification of this repulsive activity led to the characterization of a $190 \mathrm{kDa}$ molecule recognized on Western blots by anti-Slit 2 antibodies. To prove that Slits are the CP repellents, P3-P6 SVZ explants from actin-GFP transgenic mice were cocultured in collagen gels facing CP from E15 to P5 wild-type mice, Slit1, or Slit2 heterozygous or homozygous mice. As described previously, cell migration of the SVZ explants was
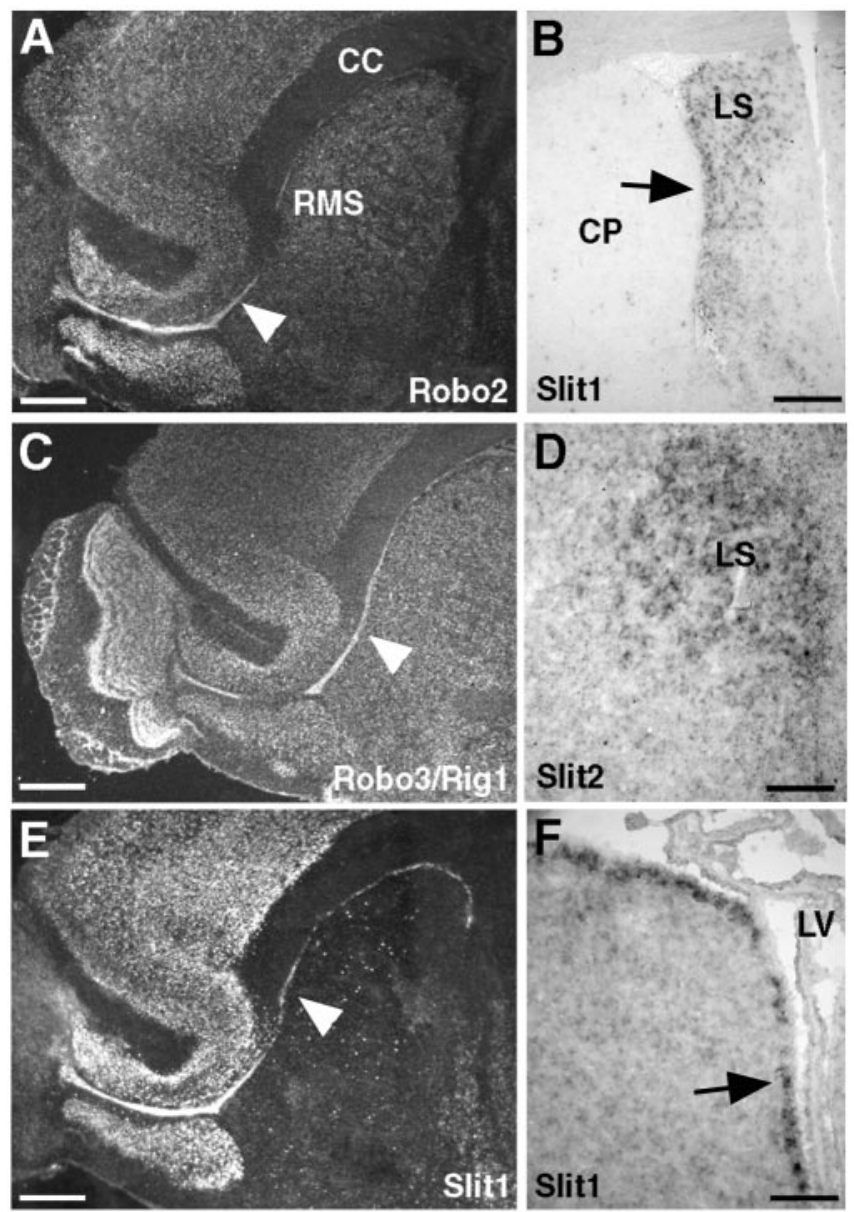

Figure 2. Expression of Slits and Robos in the adult RMS and septum. Coronal $(B, F)$ and sagittal $(A, C-E)$ sections were hybridized with digoxygenin-labeled riboprobes $(B, D, F)$ or ${ }^{35}$ S-labeled riboprobes $(A, C, E)$. Robo2 $(A)$ and Robo3/Rig-1 ( () mRNAs are highly expressed in the RMS (arrowheads). Slit1 ( $B$ ) and Slit2 ( $D$ ) are detected in the lateral septum ( $B$, arrow). Slit1 is also expressed in cells in the SVZ ( $F$, arrow) and RMS (E, arrowhead). CC, Corpus callosum; $C P$, caudate putamen; $\mathrm{LS}$, lateral septum; $\mathrm{LV}$, lateral ventricle; $\mathrm{RMS}$, rostral migratory stream. Scale bars: $A, C, E, 500 \mu \mathrm{m} ; B, D, F, 100 \mu \mathrm{m}$.

asymmetrical when cocultured with wild-type $\mathrm{CP}(\mathrm{Hu}, 1999)$ (Fig. 1 E) $(0.525 \pm 7.5, \mathrm{P} / \mathrm{D}$ ratio \pm SEM from six independent experiments). The repulsive activity was not significantly different with CP explants dissected from Slit1-/ - mice (Fig. 1D) $(0.548 \pm 5)$. In contrast, when confronted with CP from Slit2-/- mice, the repulsive activity was significantly abolished (Fig. $1 F)(0.753 \pm 4.3 ; p<0.01)$. Thus, Slit2 is the choroid plexus-derived chemorepulsive factor.

\section{Expression pattern of Slits and Robos in the adult SVZ and RMS}

Although Slits and Robos are considered to play a major role in orienting SVZ cell migration their expression in the septum or RMS of adult rodents has not been carefully analyzed. Thus, we performed in situ hybridization on sections from adult rat and mouse brain, with riboprobes for Robo1, Robo2, Rig-1/Robo3, Slit1, Slit2, and Slit3. Robo2 and Rig-1/Robo3 transcripts were highly expressed in the SVZ and RMS (Fig. 2A,C; data not shown) whereas Robo1 was undetectable (data not shown). Another argument in favor of the actual model of Slit action was the strong expression of both Slit1 and Slit2 mRNAs in the adult septum (Fig. 2B,D). Surprisingly, Slit1 mRNA was also highly 
expressed in many cells in the SVZ (Fig. $2 F)$ and all along the RMS (Fig. $2 E$ ).

Slit 1 is expressed by type $C$ cells and migrating neuroblasts

In the absence of anti-Slit1 antibodies, we tried to confirm this observation by analyzing GFP expression in adult Slit1mutant mice. These animals were generated with an internal ribosomal entry site element between the Slit1 promoter and a tau-GFP reporter gene (Plump et al., 2002). Indeed, during the first two postnatal weeks, GFP expression in Slit1+/mice closely reflected the expression of the Slit1 mRNA, as previously shown at embryonic stages (Nguyen-Ba-Charvet et al., 2002). In the adult brain and in agreement with the in situ hybridization data, GFP was still highly expressed in the SVZ, and all along the RMS from de SVZ to the olfactory bulb (Fig. 3A). The primary olfactory axons and a subset of vomeronasal axons were also labeled (Fig. 3A). These were the only regions of the adult CNS were GFP was detectable. Although Slit1 mRNA was detected in the neocortex (Fig. 2E), GFP was not expressed in this region (Fig. $3 A$ ). This suggests that either the level of expression of GFP is too low in adult cortical neurons or that they don't express the Slit1 protein. Alternatively, the regulation of the transgene expression in some neurons could differ from the endogenous protein because of the targeting strategy used to generate the mice (Plump et al., 2002) that could have modified the activity of the Slit1 promoter.

Because many cell types coexist in the SVZ and RMS (see Introduction), we tried to determine which cell types express GFP in Slit1+/- mice using multiple markers (Doetsch et al., 1997, 2002). In the RMS, GFP was systematically coexpressed with two markers of the migrating neuroblasts: mCD24 (Fig. $3 B-E$ ) and PSA-NCAM (Fig. 3F-I) (Doetsch et al., 1997). In contrast, in the SVZ, although Slit1/GFP was also colocalized with PSA-NCAM (Fig. 4A-C) and non-ependymal mCD24 (data not shown), many cells were GFP-positive, but mCD24- and PSA-NCAM-negative. The latter were grouped in clusters in continuity with chains of type A cells (Fig. 4C). This suggested that Slit1/GFP was also expressed by type C cells. Recently, Dlx2 was shown to be expressed by $\mathrm{C}$ cells and migrating neuroblasts (Doetsch et al., 2002). We observed that Dlx2-positive cells were also labeled with GFP supporting our hypothesis (Fig. 4D-F). To further characterize these cells, the cell division marker BrdU was injected $3 \mathrm{hr}$ before perfusion. All BrdU-positive cells in the SVZ were double-labeled with GFP (Fig. 4G-I), confirming the expression of Slit1 by type C cells. In the SVZ, type B cells are immunoreactive for GFAP and we did not find any colocalization of GFP with GFAP in the SVZ (Fig. $4 J-L$ ). Likewise, in the RMS, GFP was not expressed in $\mathrm{GFAP}^{+}$cells that surround migrating neuroblasts (Fig. 4M). Last, GFP was not detected in mCD24positive ependymal cells (data not shown). Therefore, all these results suggest that Slit1 is only expressed in type A and C cells (Fig. $4 N)$.

\section{Migration defects in adult Slit1 null mutant mice}

Slit2-/- mice and Slit1/Slit2 double mutant mice die at birth but Slit $1-/-$ mice are viable and fertile (Plump et al., 2002). Thus, the organization and properties of the adult SVZ and RMS could only be studied in mice lacking Slit1. As previously described in wild-type mice, chains of GFP/PSA/mCD24-expressing type A cells were exclusively observed in the RMS rostral to the SVZ in adult Slit $1+/-$ mice (Fig. 3). In contrast, in Slit $1-/-$ mice, in addition to the RMS chains, small chains of migrating GFP cells were found caudal to the SVZ, throughout the corpus callosum (Fig. 5D,G,J). We counted an average of $17.5 \pm 5$ cells per section of homozygous forebrain, for only $1.9 \pm 1$ cells on sections of heterozygous mice. Most GFP-positive cells were still found in the RMS. Many of these caudally migrating cells were proliferating cells as shown by the presence of Ki67 protein, a marker of proliferation in the initial phase of adult neurogenesis (Fig. 5A, $B$ ) (Kee et al., 2002) and BrdU (Fig. 5F-H). In addition, these caudal cells were also immunostained with PSA-NCAM (Fig. 5C,E) but not GFAP (Fig. 5I,K). Thus the caudal cells in the Slit1 -/ - mice have all characteristics of type A and type $\mathrm{C}$ cells that are normally restricted to the SVZ and RMS. Most GFP-positive cells were still found in the RMS. However, we also observed in the Slit1 mutant many GFP-positive cells leaving the RMS prematurely to migrate dorsally (Fig. 5B). 

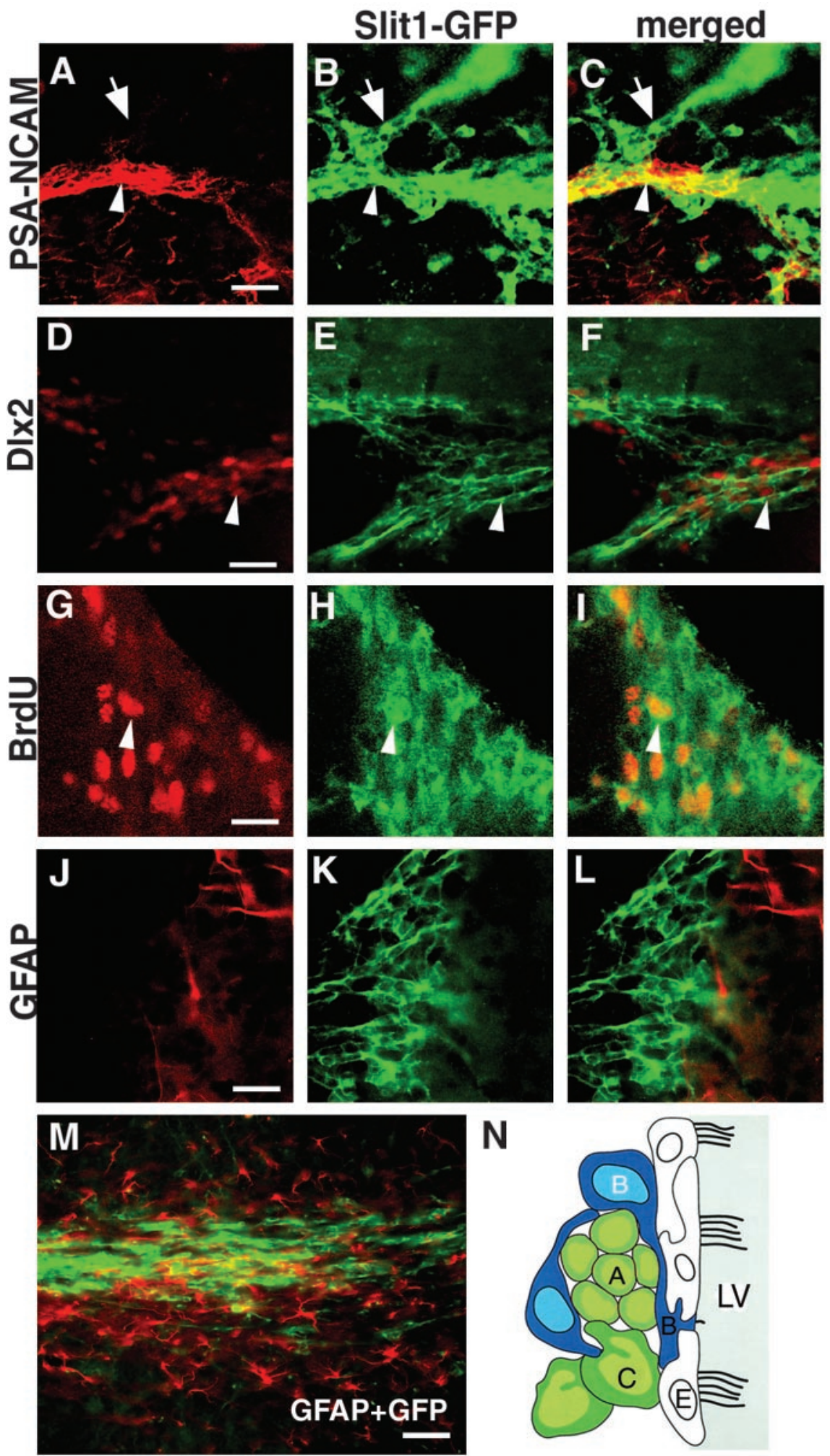

Figure 4. Slit1/GFP is expressed by type $A$ and type $C$ cells. Sagittal $(A-F, J-M)$ or coronal $(G-l)$ sections of adult SVZ were immunostained for GFP (green) and markers of the different SVZ cell types (red). A-C, Many Slit1/GFP-labeled cells also express PSA-NCAM (arrowheads) and can be identified as migrating type A neuroblasts. Clusters of PSA-NCAM-negative cells (arrow) are in continuity to type A cells. D-F, Type ( cells and migrating neuroblasts express Dlx2 (red) and are double-labeled (arrowhead) with Slit1/GFP (green). G-I, Confocal optical sections of the SVZ from mice treated with the BrdU short survival protocol. All the $\mathrm{BrdU}^{+}$cells (type A and (cells) coexpress Slit1/GFP (arrowhead). J-L, Slit1/GFP-positive cells in the SVZ (J) are not GFAP positive (type B cells, K, L). M, In the RMS, Slit1/GFP is not colocalized with GFAP. N, Schematic representation of SVZ cellular architecture modified from Alvarez-Buylla and Garcia-Verdugo (2002). The ventricular cavity would be to the right of the ependymal cell layer (white). Slit1/GFP (green) is expressed by migrating neuroblasts (type A cells) and type C cells. These cells are in intimate contact with type B cells (blue). Scale bars: $A, 120 \mu \mathrm{m} ; D, 110 \mu \mathrm{m} ; G, J, 85 \mu \mathrm{m} ; J, 95 \mu \mathrm{m} ; M, 100 \mu \mathrm{m}$.
Normal progenitor proliferation in adult Slit1-/- mice

SVZ cells can be characterized by their proliferation rate (Doetsch et al., 1999b). Type $\mathrm{A}$ and $\mathrm{C}$ cells are rapidly dividing cells, whereas type B cells proliferate much more slowly. We used the cell division marker BrdU to analyze the different proliferating cell populations in the wild-type and Slit1-mutant SVZ and RMS. To characterize the rapidly dividing cell population in the SVZ and RMS, a single injection was given $3 \mathrm{hr}$ before perfusion (see Materials and Methods). We selected seven rostrocaudal levels along the RMS, as illustrated in Figure $6 B$ from the core of the olfactory bulb to the caudal part of the lateral ventricle. In the wild-type as well as in Slit1-deficient mice, BrdU-labeled cells were found in the SVZ and all along the RMS (Fig. 6C; data not shown). We counted the number of BrdU-labeled cells at all these seven levels. We did not detect any difference of number of $\mathrm{BrdU}^{+}$cells between the three genotypes, at any level (Fig. 6A) ( $p>0.05$ in ANOVA). Therefore, Slit1 deletion does not affect rapid cell proliferation in the SVZ or the RMS.

Type B cells are slowly proliferating astrocytes. We examined whether this cell population was affected by Slit1 inactivation. To label them, we used the same protocol as Doetsch et al. (1999b), in which BrdU was administered continuously in the mice drinking water for 2 weeks, followed by a 1 week chase period without BrdU. Therefore, only slow proliferating cells retained the marker. As described previously for wild-type mice (Doetsch et al., 1999b; Johansson et al., 1999), numerous BrdU-labeled cells were found in the subventricular zone, often grouped in clusters. Next, the number of $\mathrm{BrdU}^{+}$cells between Slit1 +/- and Slit1-/- mice was compared. Every $\mathrm{BrdU}^{+}$cells were counted from randomly taken photographs of the lateral ventricle (Fig. 6D) (Slit1+/ $n=13$ mice; Slit1 $-/-n=9$ ). No significant difference was observed between the two groups $(p=0.3$ in ANOVA). This result indicates that Slit1 inactivation does not influence slow neural cell proliferation in the adult SVZ.

\section{PSA-NCAM $^{+}$cells lose Slit1/GFP} expression when migrating radially in the olfactory bulb

Once migrating neuroblasts enter the olfactory bulb, they switch from tangential chain migration to radial migration to reach their appropriate layer and differentiate into granule or periglomerular interneurons. Therefore, it was interesting to 
study more carefully Slit1/GFP expression at this strategic location. As reported above, in the RMS, all PSA-NCAM ${ }^{+}$cells were also Slit1/GFP ${ }^{+}$. In contrast, when we analyzed radially migrating $\mathrm{OB}$ neurons, we found mostly cells single-labeled with Slit1/GFP or PSA-NCAM (Fig. 7A$F)$. Thus, the shift from tangential chain migration to individual radial migration is accompanied by a variation of PSANCAM or Slit1/GFP expression in most olfactory bulb interneurons.

Abnormal cell migration without Slit1

To investigate the potential function of Slit1 on SVZ cell migration, spheres generated from SVZ of P0-P3 Slit1+/- or Slit $1-/-$ mice were plated on a mix of poly-L-ornithine/collagen substrate to compare their migratory behavior. The migration distance was evaluated after 5 DIV. Cells migrating from Slit1-/- neurospheres were found to migrate further than cells from Slit1+/- neurospheres, whether cultured in a medium with or without EGF and FGF (nef or n, respectively) (Fig. 8A). Moreover, two modes of cell migration could be distinguished: some display a classic chain migration (Fig. 8D-F), as described in vivo (Lois et al., 1996) and in vitro for SVZ explants in matrigel (Wichterle et al., 1997), whereas the others migrated in a more dispersed way (Fig. $8 C, E)$. Half $(51 \pm 14.8 \%)$ of the Slit $1+/-$ neurosphere cells migrated in chain, whereas the other half $(49 \pm 14.8 \%)$ migrated in the dispersed way (Fig. $8 \mathrm{~B}$ ). In contrast, a majority of Slit $1-/-$ cells migrated according to the dispersed way $(70.9 \pm 4.7 \%)$ (Fig. 8 B). Thus, Slit1 inactivation alters the migration of neurosphere-derived cells.

These data showed that Slit1 inactivation influences neurosphere migration. We therefore asked whether the absence of Slit 1 could also affect neuronal migration from SVZ explants cultured in matrigel, a three-dimensional artificial matrix, which permits chain migration of neuroblasts in vitro (Wichterle et al., 1997). Explants from Slit1 $+/-$ and Slit1-/- mice were cultured for $4 \mathrm{~d}$ (Fig. 9A-C), and two parameters were quantified. First, the migration distance was analyzed by performing for each explant, 24 measurements between the edge of the explant and the migration front. As observed with neurospheres, there was a significant increase of the migration distance from Slit1 $-/-$ explants compared with the Slit1+/- explants (Fig. 9D) (Slit1+/-, $n=18$; Slit1 $-/-, n=18 ; p<0.0001$ in ANOVA). Slit1+/explants showed an average migration distance of $602 \pm 12 \mu \mathrm{m}$, whereas Slit $1-/-$ migrate at $713 \pm 12 \mu \mathrm{m}$. We also quantified $\mu \mathrm{m}$.
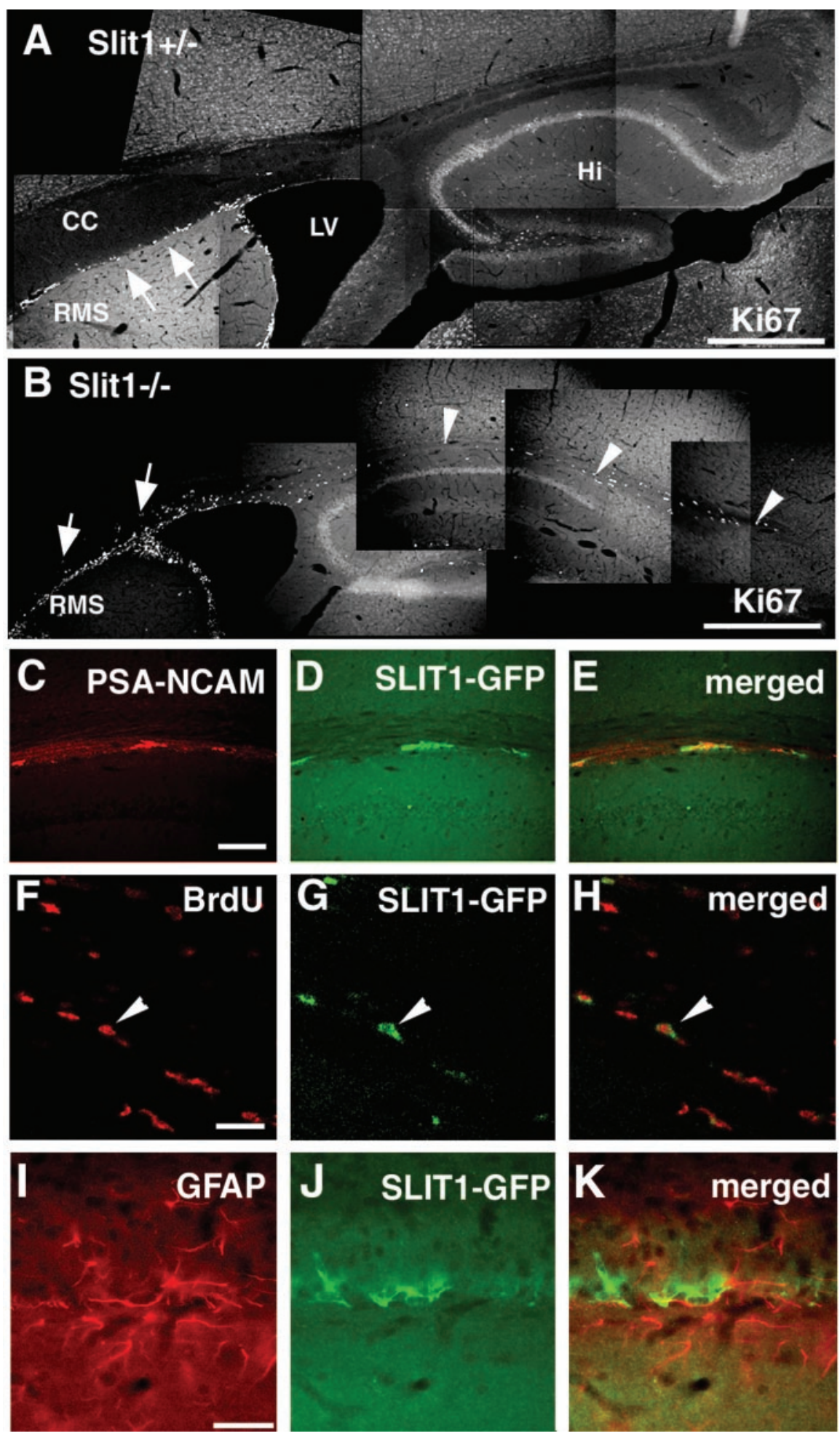

Figure 5. Abnormal neuroblast migration in Slit1-deficient mice. Sagittal sections of Slit1 $+/-(A)$ and Slit1- - mice $(B-K) . A$, In Slit1 + / - mice, all Ki67 + cells deriving from the SVZ migrate rostrally within the RMS (arrows). B, in Slit1 - / mice a majority of Ki67 + cells are also found in the RMS, but many Ki67 ${ }^{+}$cells migrate caudally in the corpus callosum, above the hippocampus ( $B$, arrowheads). Some are also found dorsal to the RMS ( $B$, arrows). These proliferating cells expressed Slit $1 / G F P(D$, $G, J)$ and are also labeled with PSA-CAM $(E)$ and $\operatorname{BrdU}(H)$ but not GFAP (K). Scale bars: $A, B, 1 \mathrm{~mm} ; C, 160 \mu \mathrm{m} ; F, 110 \mu \mathrm{m} ; I, 270$

the number of cells migrating over $600 \mu \mathrm{m}$. We observed an significant increase ( $p<0.05$ in ANOVA) of almost $400 \%$ between Slit $1+/-(45.2 \pm 7.2$ cells per explant; $n=18)$ and Slit1-/- (219 \pm 79 cells per explant; $n=18)$. Second, we 
A
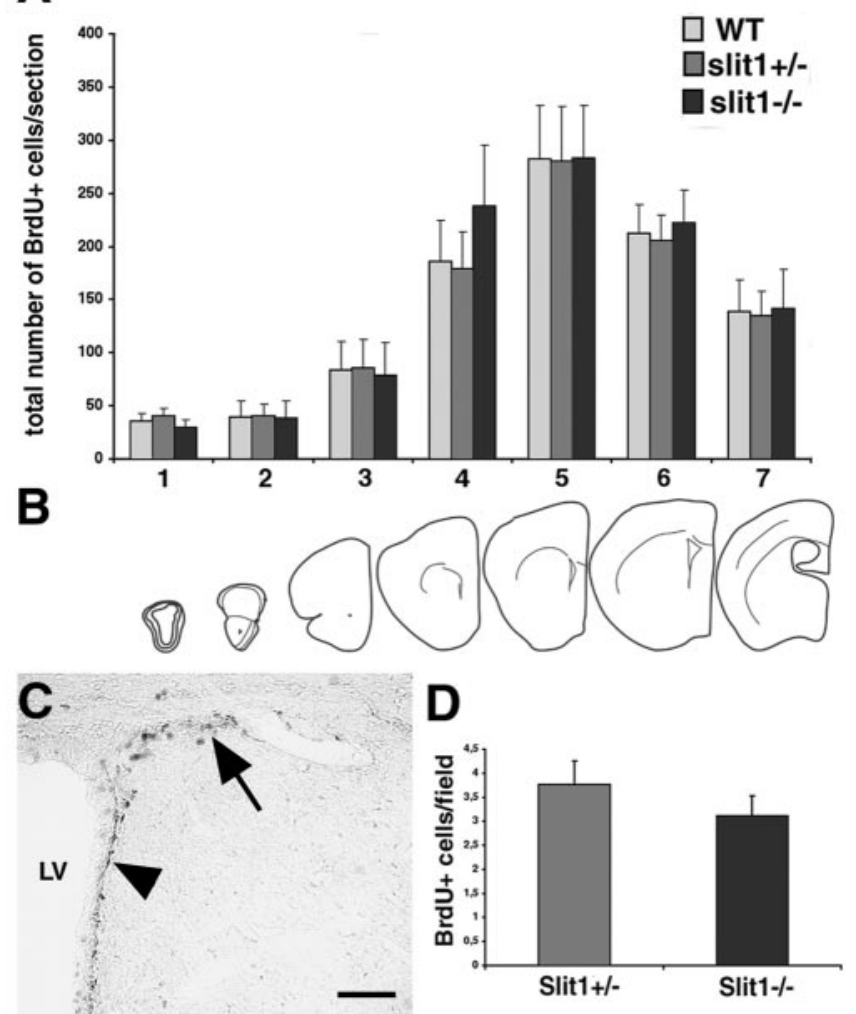

Figure 6. Normal neuronal proliferation in adult Slit1-deficient mice. $A$, Comparison of rapid cell proliferation of wild type (WT), Slit1+/-, and Slit1-/- mice. The number of $\mathrm{BrdU}^{+}$ cells was counted at different levels, from rostral (left) to caudal (right) ( $B$ ) of the RMS when the animals were treated with the short survival protocol $(A)$. No significant difference was detected. C, A coronal section showing BrdU-positive cells in the SVZ (arrowhead) and the RMS (arrow) of a Slit1-/- mice. D, Comparison of slow cell proliferation in Slit1 $+/-$ and Slit1 - / - mice. No significant difference in the number of BrdU ${ }^{+}$cells was detected in mice treated with the long survival protocol. Scale bar, $50 \mu \mathrm{m}$.
PSA-NCAM
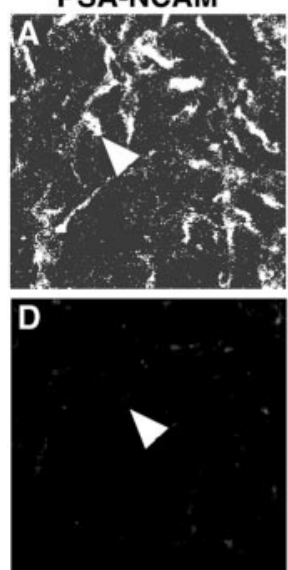

Slit1-GFP
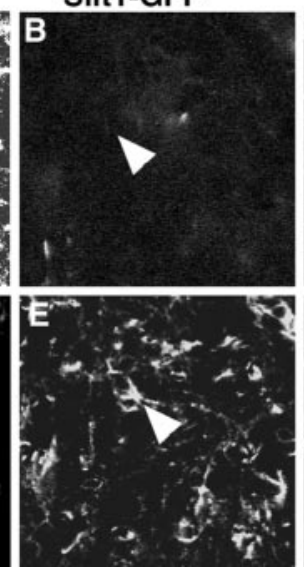

merged
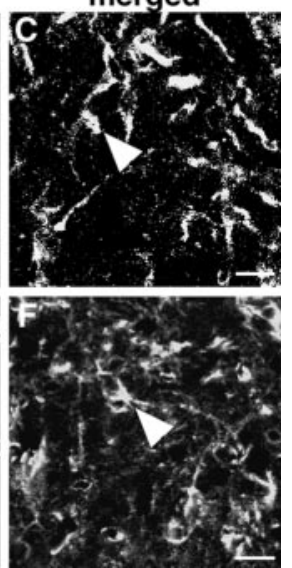

Figure 7. Regulation of Slit1/GFP expression in the olfactory bulb. Once in the OB, chainmigrating neuroblasts have to migrate radially to reach their final location. In Slit1 mice, PSA$\mathrm{NCAM}^{+}$neurons are no longer double-labeled with GFP when they start migrating radially in the olfactory bulb. $A-C$ are confocal optical sections of radially migrating $O B$ neurons immunostained for PSA-NCAM $(A)$ and GFP $(B)$ showing that PSA-NCAM + cells (arrowhead) do not coexpress GFP. $D-F$ are confocal optical sections of the same region than in $A-C$ but at a different $z$ level, showing that Slit1/GFP + cells (arrowhead) are not expressing PSA-NCAM. Scale bar, $75 \mu \mathrm{m}$.
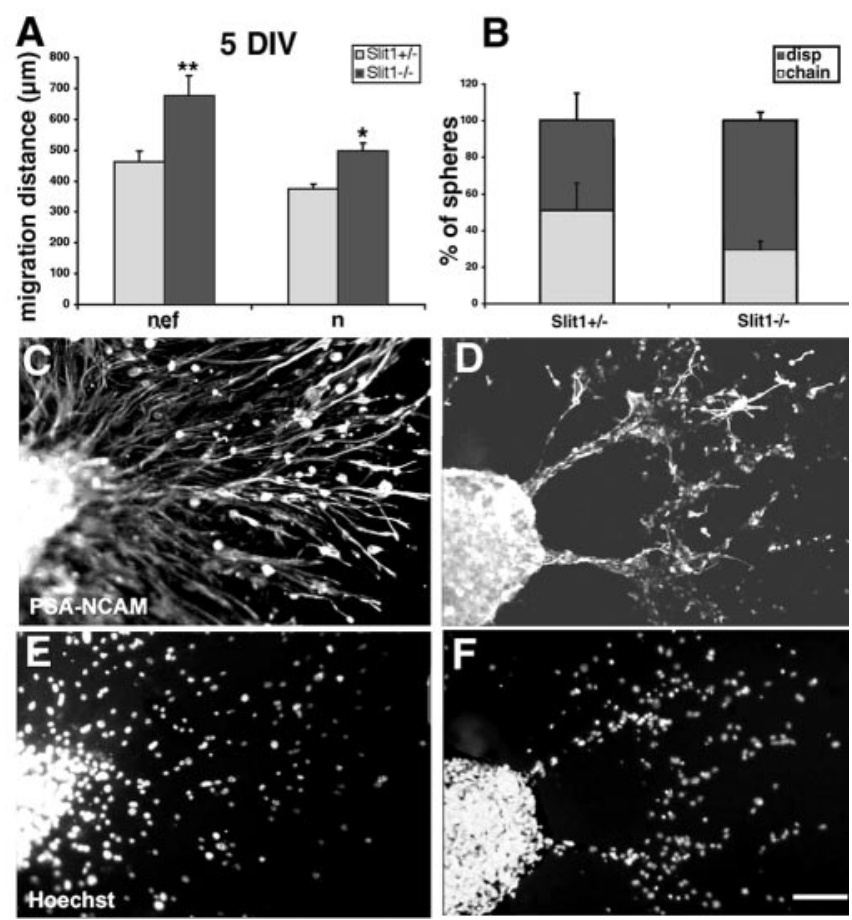

Figure 8. Abnormal neuronal migration from neurospheres lacking Slit1. $A$, Neurospheres obtained from Slit1 +/ - or Slit1 - / - newborn mice were seeded on poly-ornithine/collagen for $5 \mathrm{~d}$ in medium containing or not EGF and FGF-2 (nef and n, respectively). In the absence of Slit1 the migration is enhanced in both conditions. B, Although Slit1 + / - cells equally migrate according to a dispersed $(C, E)$ or a chain-like pattern $(D, F)$, Slit $1-/-$ cells preferentially adopt a dispersed pattern of migration. Scale bar: $\left(-F, 50 \mu \mathrm{m} .{ }^{*} p<0.05 ;{ }^{* *} p<0.001\right.$.
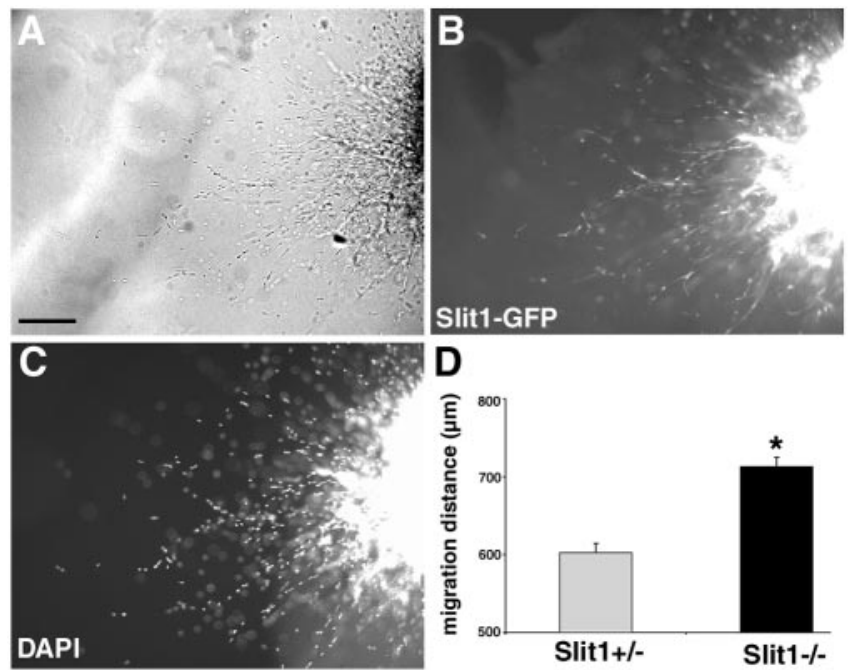

Figure 9. Abnormal neuronal migration from SVZ explants lacking slit1. Explants from Slit7deficient mice were cultured $4 \mathrm{~d}$ in vitro in matrigel $(A)$, immunostained with GFP $(B)$, and counterstained with $\operatorname{DAPI}(C) . D$, The quantification of migration distance reveals a significant increase of migration distance $(p<0.0001)$ with SVZ explants from Slit1 - / - mice. Scale bar, $120 \mu \mathrm{m}$.

counted individual cells from randomly taken photographs of Slit1 $+/-$ and Slit1 $-/-$ explants. No significant difference was observed between the two groups (Slit1+/-, $n=18$; Slit1-/-, $n=18 ; p<0.4$ in ANOVA; data not shown). Altogether, these results suggest that Slit1 controls the migration of SVZ neuroblasts cell autonomously. 


\section{Discussion}

Retrovirus-mediated gene transfer (Luskin, 1993; Petreanu and Alvarez-Buylla, 2002), transplantation studies ( $\mathrm{Hu}$ and Rutishauser, 1996; Jankovski and Sotelo, 1996) and cell labeling experiments (Luskin, 1993; Doetsch and Alvarez-Buylla, 1996) among others have revealed the unidirectional migration of postnatal SVZ neuroblasts along the RMS. The corollary is that guidance molecules able to orient the migration of these cells from the SVZ to the olfactory bulb must exist, in particular in the adult telencephalon. The first evidence for their existence came from the analysis of organotypic cultures in three-dimensional gels (Hu and Rutishauser, 1996), revealing that the caudal septum secretes diffusible repulsive factors for olfactory interneuron precursor cells. Likewise, the choroid plexus of the lateral ventricle has also been shown to produce similar chemorepellents $(\mathrm{Hu}$, 1999). Some recent results suggest that chemorepulsion might not be the sole guidance mechanism as the olfactory bulb, the final target of the SVZ neuroblasts, appears to secrete chemoattractant for these cells (Liu and Rao, 2003). However, previous in vitro experiments ( $\mathrm{Hu}$ and Rutishauser, 1996; Wichterle et al., 1997) and olfactory bulb ablation studies (Jankovski et al., 1998; Kirschenbaum et al., 1999) raise doubt on the role of these attractants in vivo. The emerging picture is that SVZ neuroblasts move forward along the RMS under the combined action of repellents and attractants. In this context the diffusible proteins Slit $1 / 2$ were suggested to play a major role in the orientation of the migration of olfactory bulb interneuron precursors $(\mathrm{Hu}$, 1999; Wu et al., 1999).

Here, we show that septum and choroid plexus from Slit1and Slit2-deficient mice have lost most, if not all, its repulsive activity. This result demonstrates that the septum and choroid plexus-derived chemorepulsive factor for migrating SVZ cells are Slits, as previously shown for the axons of olfactory bulb projection neurons (Nguyen-Ba-Charvet et al., 2002). However, the persistence in the adult brain of a Slit-mediated repulsion of SVZ neuroblasts remained hypothetical. In this respect, we found that in adult mice, the expression of Slit1 and Slit2 is maintained in the adult septum and that type A cells express high levels of Robo2 and Robo3/Rig-1 receptors during their migration in the RMS (Marillat et al., 2002). Thus, Slit/Robo chemorepulsion is likely to occur in the adult RMS. Our results also show that in adult mice lacking Slit1, chains of cells with phenotypical characteristics of SVZ neuroblasts migrate rostrally along the RMS but also caudally to the splenium of the corpus callosum. Thus, in Slit1 null mutant mice tangential migration is not unidirectional as in wildtype mice but bidirectional. These data are in favor of the existing, purely repulsive, model of action of Slits on SVZ neuroblasts.

\section{Cell autonomous versus non-cell autonomous control of neuronal migration by Slit1}

The exact mechanism of action of Slits on migrating SVZ cells is still controversial. Slits were proposed to be inhibitors of migration rather than repellents (Mason et al., 2001). More recently, elegant in vitro assays have provided direct evidence for the repulsive activity of Slits on SVZ neuroblasts (Ward et al., 2003). However, these results are difficult to compare because one group of investigators used Slit2-secreting cells facing neuroblasts from the most anterior part of the RMS and twodimensional cultures (Mason et al., 2001), whereas another used Slit1-expressing cells and SVZ explants in collagen-matrigel matrices (Ward et al., 2003). The presence of caudal neuronal chains in Slit1-deficient mice supports the repulsion hypothesis. However, the finding that most SVZ cells continue to migrate in the right direction (toward the olfactory bulb) along the RMS in the Slit1-/ - mice indicate that Slit1 is not essential for the migration and/or orientation. The fact that only some cells migrate caudally could be explained if the most important role of Slit1 is to prevent the SVZ cells from entering the wrong areas on the brain (e.g., the corpus callosum). Furthermore, the strong expression of Slit 1 on type $\mathrm{A}$ and type $\mathrm{C}$ cells indicates that the purely repulsive model is not fully satisfactory.

How can Slits repel neuroblasts that express themselves high levels of Slits? If Slit1 were only an inhibitor, then Roboexpressing neuroblasts should not be able to migrate in the RMS. The analysis of Slits and Robos expression pattern (Marillat et al., 2002) has shown that many Robo-expressing neurons can also express Slits or are located in regions that express Slits. Our results confirm these data and strongly suggest that Slit1 has a paracrine or autocrine action. We show that cells migrating from Slit1-mutant neurospheres migrate farther than cells from wildtype mice and adopt a more dispersed mode of migration and that in Slit1-deficient mice SVZ-derived cells leave the RMS prematurely. This suggests that Slit1 expression in migrating SVZ cells is important to maintain neuronal chain integrity, probably to keep cells in the pathway, but also that Slit1 might slow down or inhibit migration cell autonomously. Other studies have shown that Slit1 and Slit2 can slowdown migration when presented to SVZ cells in a uniform concentration (Mason et al., 2001; Ward et al., 2003). In addition, Slit1 and Slit2 expression is changed in many metastatic tumor cells, and Slits might be able to restrain their migration (Dallol et al., 2003; Wang et al., 2003). This suggests that Slit1 could act both as a long-range factor, which together with Slit2 would give their initial orientation to migrating SVZ neuroblasts, and also as a short-range factor helping the construction of the migrating chains. In vitro, Slits only act at a maximum distance of $1 \mathrm{~mm}$ (Wu et al., 1999), whereas the adult RMS is several millimeters long. Having Slit secreted all along the RMS might help maintaining the gradient of repellent up to the olfactory bulb. This dual short-range/long-range function of Slit1 could be related to the biochemical properties of these proteins. Slits, although secreted, are known to be highly charged and tightly bound to cell membrane (Nguyen-BaCharvet et al., 2001). Interestingly, soluble and immobilized Slit does not seem to have the same activity on axons and neurons (Nguyen-Ba-Charvet et al., 2001; De Bellard et al., 2003).

The chains of migrating cells and their astrocytic channels in the RMS are one of the only regions in the brain devoid of axons in normal condition (Jankovski and Sotelo, 1996; Doetsch et al., 1997). Because Slit1 is repellent for many telencephalic axons (Nguyen Ba-Charvet et al., 1999; Shu and Richards, 2001), one additional important function of having high Slit expression within the RMS could be to keep axons away from the pathway and thus to facilitate neuronal migration by creating an exclusion zone. The cells that migrate dorsally, out of the RMS in the Slit1 mutant mice (Fig. $5 B$ ) are in favor of this hypothesis. We also observed that cells migrating out of Slit $1-/$ - neurospheres seem to lose their ability to migrate in chains.

\section{Is Slit1 function in neuronal migration modulated by other factors?}

Modelizing Slit function is particularly complex as, in addition to binding Robos, Slits were shown to interact with many other molecules (Brose et al., 1999; Liang et al., 1999; Hu, 2001; Ronca et al., 2001). Slit can bind the secreted factor Netrin-1, whose receptor DCC is also expressed in RMS neuroblasts (Brose et al., 1999; Murase and Horwitz, 2002). In other systems, Slits were 
shown to modulate the response of growing axons (Stein and Tessier-Lavigne, 2001) or migrating neurons (Causeret et al., 2002) by silencing Netrin-1 signaling. The presence of high concentration of Slits around migrating SVZ neuroblasts could have a similar function.

We found that Slit1 expression in migrating olfactory bulb interneuron precursors is rapidly downregulated as they reach the olfactory bulb. This change of expression precisely corresponds to the moment migrating olfactory bulb precursors switch from a tangential chain migration to an individual radial migration (Rousselot et al., 1995) and change their expression of NMDA receptors (Carleton et al., 2003). Recently, it has been shown that the extracellular matrix molecule Reelin acts as a dispersal signal for SVZ neuroblasts inducing them to stop migrating in chains (Hack et al., 2002). Reelin function is probably to change adhesive interactions between precursors, thus facilitating their detachment from the chains. The results of the neurosphere cultures show that SVZ cells lacking Slit1 migrate preferentially independently. This suggests that Slit1 could be one of the targets of Reelin.

In conclusion, our results show that Slit function in the regulation of the migration of SVZ neuroblasts is highly complex: these molecules are not only repellents and can act autonomously. It will be important to determine the fate of caudal migrating cells, because we did not observe them outside the corpus callosum.

\section{References}

Alvarez-Buylla A, Garcia-Verdugo JM (2002) Neurogenesis in adult subventricular zone. J Neurosci 22:629-634.

Belvindrah R, Rougon G, Chazal G (2002) Increased neurogenesis in adult mCD24-deficient mice. J Neurosci 22:3594-3607.

Brose K, Bland KS, Wang K-H, Arnott D, Henzel W, Goodman CS, TessierLavigne M, Kidd T (1999) Slit proteins bind Robo receptors and have an evolutionarily conserved role in repulsive axon guidance. Cell 96:795-806.

Carleton A, Petreanu LT, Lansford R, Alvarez-Buylla A, Lledo PM (2003) Becoming a new neuron in the adult olfactory bulb. Nat Neurosci 6:507-518.

Causeret F, Danne F, Ezan F, Sotelo C, Bloch-Gallego E (2002) Slit antagonizes netrin-1 attractive effects during the migration of inferior olivary neurons. Dev Biol 246:429-440.

Conover JC, Doetsch F, Garcia-Verdugo JM, Gale NW, Yancopoulos GD, Alvarez-Buylla A (2000) Disruption of Eph/ephrin signaling affects migration and proliferation in the adult subventricular zone. Nat Neurosci 3:1091-1097.

Dallol A, Morton D, Maher ER, Latif F (2003) SLIT2 axon guidance molecule is frequently inactivated in colorectal cancer and suppresses growth of colorectal carcinoma cells. Cancer Res 63:1054-1058.

De Bellard ME, Rao Y, Bronner-Fraser M (2003) Dual function of Slit2 in repulsion and enhanced migration of trunk, but not vagal, neural crest cells. J Cell Biol 162:269-279.

Decker L, Avellana-Adalid V, Nait-Oumesmar B, Durbec P, Baron-Van Evercooren A (2000) Oligodendrocyte precursor migration and differentiation: combined effects of PSA residues, growth factors, and substrates. Mol Cell Neurosci 16:422-439.

Doetsch F, Alvarez-Buylla A (1996) Network of tangential pathways for neuronal migration in adult mammalian brain. Proc Natl Acad Sci USA 93:14895-14900.

Doetsch F, Garcia-Verdugo JM, Alvarez-Buylla A (1997) Cellular composition and three-dimensional organisation of the subventricular germinal zone in the adult mammalian brain. J Neurosci 17:5046-5061.

Doetsch F, Garcia-Verdugo JM, Alvarez-Buylla A (1999a) Regeneration of a germinal layer in the adult mammalian brain. Proc Natl Acad Sci USA 96:11619-11624.

Doetsch F, Caille I, Lim DA, Garcia-Verdugo JM, Alvarez-Buylla A (1999b) Subventricular zone astrocytes are neural stem cells in the adult mammalian brain. Cell 97:703-716.
Doetsch F, Petreanu L, Caille I, Garcia-Verdugo JM, Alvarez-Buylla A (2002) EGF converts transit-amplifying neurogenic precursors in the adult brain into multipotent stem cells. Neuron 36:1021-1034.

Gage FH, Ray J, Fisher LJ (1995) Isolation, characterization, and use of stem cells from the CNS. Annu Rev Neurosci 18:159-192.

Hack I, Bancila M, Loulier K, Carroll P, Cremer H (2002) Reelin is a detachment signal in tangential chain-migration during postnatal neurogenesis. Nat Neurosci 5:939-945.

Hadjantonakis A-K, Gertsenstein M, Ikawa M, Okabe M, Nagy A (1998) Generating green fluorescent mice by germline transmission of green fluorescent ES cells. Mech Dev 76:79-90.

Hu H (1999) Chemorepulsion of neuronal migration by slit2 in the developing mammalian forebrain. Neuron 23:703-711.

$\mathrm{Hu} \mathrm{H}$ (2000) Polysialic acid regulates chain formation by migrating olfactory interneuron precursors. J Neurosci Res 61:480-492.

$\mathrm{Hu} \mathrm{H}$ (2001) Cell-surface heparan sulfate is involved in the repulsive guidance activities of Slit2 protein. Nat Neurosci 4:695-701.

$\mathrm{Hu} \mathrm{H}$, Rutishauser U (1996) A septum-derived chemorepulsive factor for migrating olfactory interneuron precursors. Neuron 16:933-940.

Hu H, Tomasiewicz H, Magnuson T, Rutishauser U (1996) The role of polysialic acid in migration of olfactory bulb interneuron precursors in the subventricular zone. Neuron 16:735-743.

Jankovski A, Sotelo C (1996) Subventricular zone-olfactory bulb migratory pathway in the adult mouse: cellular composition and specificity as determined by heterochronic and heterotopic transplantation. J Comp Neurol 371:376-396.

Jankovski A, Garcia C, Soriano E, Sotelo C (1998) Proliferation, migration and differentiation of neuronal progenitor cells in the adult mouse subventricular zone surgically separated from its olfactory bulb. Eur J Neurosci 10:3853-3868.

Johansson CB, Momma S, Clarke DL, Risling M, Lendhal U, Frisen J (1999) Identification of a neural stem cell in the adult mammalian central nervous system. Cell 96:25-34.

Kee N, Sivalingam S, Boonstra R, Wojtowicz JM (2002) The utility of Ki-67 and BrdU as proliferative markers of adult neurogenesis. J Neurosci Methods 115:97-105.

Kirschenbaum B, Doetsch F, Lois C, Alvarez-Buylla A (1999) Adult subventricular zone neuronal precursors continue to proliferate and migrate in the absence of the olfactory bulb. J Neurosci 19:2171-2180.

Liang Y, Annan RS, Carr SA, Popp S, Mevissen M, Margolis RK, Margolis RU (1999) Mammalian homologues of the Drosophila slit protein are ligands of the heparan sulfate proteoglycan glypican-1 in brain. J Biol Chem 274:17885-17892.

Liu G, Rao Y (2003) Neuronal migration from the forebrain to the olfactory bulb requires a new attractant persistent in the olfactory bulb. J Neurosci 23:6651-6659.

Lois C, Alvarez-Buylla A (1993) Proliferating subventricular zone cells in the adult mammalian forebrain can differentiate into neurons and glia. Proc Natl Acad Sci USA 90:2074-2077.

Lois C, Alvarez-Buylla A (1994) Long-distance neuronal migration in the adult mammalian brain. Science 264:1145-1148.

Lois C, Garcia-Verdugo JM, Alvarez-Buylla A (1996) Chain migration of neuronal precursors. Science 271:978-981.

Luskin MB (1993) Restricted proliferation and migration of postnatally generated neurons derived from the forebrain subventricular zone. Neuron 11:173-189.

Marillat V, Cases O, Nguyen-Ba-Charvet KT, Tessier-Lavigne M, Sotelo C Chédotal A (2002) Spatiotemporal expression patterns of slit and robo genes in the rat brain. J Comp Neurol 442:130-155.

Marin O, Plump AS, Flames N, Sanchez-Camacho C, Tessier-Lavigne M, Rubenstein JLR (2003) Directional guidance of interneuron migration to the cerebral cortex relies on subcortical Slit1/2-independent repulsion and cortical attraction. Development 130:1889-1901.

Mason HA, Ito S, Corfas G (2001) Extracellular signals that regulate the tangential migration of olfactory bulb neuronal precursors: inducers, inhibitors, and repellents. J Neurosci 21:7654-7663.

Murase S, Horwitz AF (2002) Deleted in colorectal carcinoma and differentially expressed integrins mediate the directional migration of neural precursors in the rostral migratory stream. J Neurosci 22:3568-3579.

Nguyen Ba-Charvet KT, Brose K, Marillat V, Kidd T, Goodman CS, TessierLavigne M, Sotelo C, Chédotal A (1999) Slit2-mediated chemorepulsion and collapse of developing forebrain axons. Neuron 22:463-473. 
Nguyen-Ba-Charvet KT, Brose K, Marillat V, Sotelo C, Tessier-Lavigne M, Chédotal A (2001) Sensory axons response to substrate-bound Slit2 is modulated by laminin and cyclicGMP. Mol Cell Neurosci 17:1048-1058.

Nguyen-Ba-Charvet KT, Plump AS, Tessier-Lavigne M, Chédotal A (2002) Slit1 and Slit2 proteins control the development of the lateral olfactory tract. J Neurosci 22:5473-5480.

Petreanu L, Alvarez-Buylla A (2002) Maturation and death of adult-born olfactory bulb granule neurons: role of olfaction. J Neurosci 22:6106-6113.

Plump AS, Erskine L, Sabatier C, Brose K, Epstein CJ, Goodman CS, Mason C, Tessier-Lavigne M (2002) Slit1 and Slit2 cooperate to prevent premature midline crossing of retinal axons in the mouse visual system. Neuron 33:219-232.

Ronca F, Andersen JS, Paech V, Margolis RU (2001) Characterization of Slit protein interactions with glypican-1. J Biol Chem 276:29141-29147.

Rousselot P, Lois C, Alvarez-Buylla A (1995) Embryonic (PSA) N-CAM reveals chains of migrating neuroblasts between the lateral ventricle and the olfactory bulb of adult mice. J Comp Neurol 351:51-61.

Shu T, Richards LJ (2001) Cortical axon guidance by the glial wedge during the development of the corpus callosum. J Neurosci 21:2749-2758.
Stein E, Tessier-Lavigne M (2001) Hierarchical organization of guidance receptors: silencing of netrin attraction by slit through a Robo/DCC receptor complex. Science 291:1928-1938.

Wang B, Xiao Y, Ding BB, Zhang N, Yuan X, Gui L, Qian KX, Duan S, Chen Z, Rao Y, Geng JG (2003) Induction of tumor angiogenesis by Slit-Robo signaling and inhibition of cancer growth by blocking Robo activity. Cancer Cell 4:19-29.

Ward M, McCann C, DeWulf M, Wu JY, Rao Y (2003) Distinguishing between directional guidance and motility regulation in neuronal migration. J Neurosci 23:5170-5177.

Wichterle H, Garcia-Verdugo JM, Alvarez-Buylla A (1997) Direct evidence for homotypic, glia-independent neuronal migration. Neuron 18:779-791.

Wu W, Wong K, Chen J-h, Jiang Z-h, Dupuis S, Wu JY, Rao Y (1999) Directional guidance of neuronal migration in the olfactory system by the protein Slit. Nature 400:331-336.

Zhu Y, Li H-s, Zhou L, Wu JY, Rao Y (1999) Cellular and molecular guidance of GABAergic neuronal migration from an extracortical origin to the neocortex. Neuron 23:473-485. 